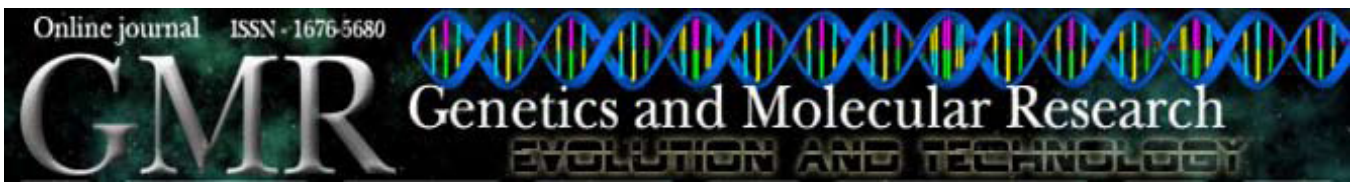

\title{
Occurrence of multiple nucleolus organizer regions and intraspecific karyotype variation in Scaptotrigona xanthotricha Moure (Hymenoptera, Meliponini)
}

\author{
O.M.P. Duarte ${ }^{1}$, C.C.C. Martins ${ }^{1}$, A.M. Waldschmidt ${ }^{2}$ and M.A. Costa ${ }^{1}$ \\ ${ }^{1}$ Departamento de Ciências Biológicas, \\ Universidade Estadual de Santa Cruz, Ilhéus, BA, Brasil \\ ${ }^{2}$ Departamento de Ciências Biológicas, \\ Universidade Estadual do Sudoeste da Bahia, Jequié, BA, Brasil \\ Corresponding author: M.A. Costa \\ E-mail: costama@uesc.br
}

Genet. Mol. Res. 8 (3): 831-839 (2009)

Received March 10, 2009

Accepted May 21, 2009

Published July 21, 2009

\begin{abstract}
Scaptotrigona xanthotricha has a wide geographic distribution in the Brazilian Atlantic rainforest. One population from southeast and two from northeast Brazil were analyzed and were found to have chromosome polymorphisms. Although the chromosome number $2 \mathrm{n}=34$ is conserved in this species, karyotypic analysis revealed clear differences between the three populations. Congruent and ubiquitous multiple nucleolus organizer regions, heterochromatin and $\mathrm{CMA}_{3}$-positive blocks were found. The variations suggest that this species is in a process of genetic differentiation. This differentiation process might have been enhanced by restricted nesting preferences, combined with recent extensive fragmentation of the
\end{abstract}


Atlantic rainforest, which limits gene flow between populations.

Key words: Chromosome; Fluorochrome staining; Ag-NOR; Stingless bee

\section{INTRODUCTION}

Interpopulational karyotype analysis in species with wide distribution can provide a framework for better understanding their origin and evolutionary history. Some examples are cytogenetic analyses carried out by Brown Jr. et al. (2004) in several species of lepidopterans of the subfamily Ithomiinae and by Mantovani et al. (2000) in the cichlid Astyanax scabripinnis.

The stingless bee Scaptotrigona xanthotricha shows a distribution ranging from the State of Paraná, Southern Brazil, to the State of Bahia, in the northeast (Moure et al., 2007). These bees build their nests inside cavities of large living trees at the understory layer of the Atlantic rainforest. Few cytogenetic studies on Scaptotrigona have been published. Only four of the 30 known species (Moure et al., 2007) were cytogenetically characterized in an extensive analysis of 15 Meliponini genera by Rocha et al. (2003). Most of these species, namely $S$. xanthotricha, $S$. depilis, and $S$. postica, are representatives of the southeast Brazilian fauna except for Scaptotrigona sp, which was collected in the State of Paraíba, in the northeast.

The numeric stability observed in the karyotypes of the tribe Meliponini, with $2 n$ $=34$ as the predominant number, indicates that the differences observed are allied to structural chromosome changes, especially in the variable heterochomatin regions along the chromosome segments (Hoshiba and Imai, 1993; Rocha et al., 2003; Costa et al., 2004). Karyotype variation produced by structural chromosome rearrangements frequently occurs in heterochromatic regions (John, 1988; Sumner, 2003). For instance, several cytogenetic studies on Hymenoptera have demonstrated the dynamic character of the heterochromatin (Imai et al., 1988; Imai, 1991, 1993).

Due to their effects on chromosome pairing and hybrid fertility, the accumulation and fixation of chromosome rearrangements can lead to the isolation of populations (John, 1988; King, 1993; Sumner, 2003). In this study, we addressed the geographic differentiation among populations of $S$. xanthotricha based on the analysis of the heterochromatin content, presence of multiple NORs (nucleolus organizer regions) and other structural karyotype variations, using different cytogenetic techniques.

\section{MATERIAL AND METHODS}

We sampled a minimum of 15 specimens from each locality (Table 1). Vouchers were deposited at the DZUP - "Coleção Entomológica Pe. J.S. Moure", Departamento de Zoologia, Universidade Federal do Paraná, Curitiba, Brazil.

Metaphasic chromosomes were obtained from cerebral ganglia of prepupae following the technique of Imai et al. (1988). We carried out C banding according to Sumner (1972). DAPI/CMA staining was carried out as described by Schweizer (1976) with modifications by Guerra and Souza (2002). Ag-NOR staining was performed according 
to Howell and Black (1980) with modifications by Kavalco and Pazza (2004), using 25\% $\mathrm{AgNO}_{3}, 1 \%$ gelatin, and $0.25 \%$ formic acid.

Table 1. Collecting locality, geographic coordinates, state, and number of specimens of Scaptotrigona xanthotricha
analyzed.
\begin{tabular}{lcccc}
\hline Locality & Geographic coordinates & Altitude (m) & State & Sample size \\
\hline Valença & $\mathrm{S} 13^{\circ} 22^{\prime} / \mathrm{W} 39^{\circ} 3^{\prime}$ & 39 & $\mathrm{BA}$ & 24 \\
Camacan & $\mathrm{S} 15^{\circ} 24^{\prime} / \mathrm{W} 39^{\circ} 2^{\prime}$ & 180 & BA & 15 \\
Viçosa & $\mathrm{S} 20^{\circ} 45^{\prime} / \mathrm{W} 42^{\circ} 52^{\prime}$ & 648 & MG & 27 \\
\hline
\end{tabular}

A minimum of 10 metaphases per specimen were analyzed. Images of NOR- and conventional-stained metaphases were captured in an Olympus BX-41 photomicroscope. Fluorescent images were captured with a Leica DMRA2 photomicroscope using the Leica IM50 software. Chromosome classification followed Imai (1991) nomenclature, based on heterochromatin distribution, in which $\mathrm{M}=$ metacentric, $\mathrm{A}=$ acrocentric, and $\mathrm{A}^{\mathrm{M}}=$ pseudoacrocentric (acrocentric with relatively long heterochromatic arm).

\section{RESULTS}

Scaptotrigona xanthotricha showed an invariable diploid chromosome number of $2 n=34$ in the females and a haploid number of $n=17$ in the males. However, we found several structural chromosome differences among the sampled populations. $\mathrm{C}$ banded karyotypes resulted in divergent karyotypic formulae; $2 \mathrm{k}=16 \mathrm{~A}+18 \mathrm{~A}^{\mathrm{M}}$ in the samples of Valença, $\mathrm{BA}, 2 \mathrm{k}=4 \mathrm{M}+12 \mathrm{~A}+18 \mathrm{~A}^{\mathrm{M}}$ in the samples of Camacan, $\mathrm{BA}$, and $2 \mathrm{k}=2 \mathrm{M}+14 \mathrm{~A}$ $+18 \mathrm{~A}^{\mathrm{M}}$ in the samples of Viçosa, $\mathrm{MG}$.

In the three populations sampled, most of the heterochromatin was distributed along the entire length of one arm in the pseudoacrocentric chromosomes (Figure 1A,B and C). In the colony from Valença, heterochromatin was also found in the short arm of eight acrocentric chromosome pairs and an additional band was observed near the centromere on the long arms of the 1st, $2 \mathrm{nd}, 3 \mathrm{rd}$, 4th, and 8th chromosome pairs. The 13 th pair showed heteromorphism in length of heterochromatic arm (Figure 1A).

In the Camacan samples, we also observed heterochromatin in the centromeric region of metacentric chromosomes, in the short arms of acrocentric chromosomes and in the pericentromeric region of the 4th, 5th, and 8th chromosome pairs (Figure 1B). Viçosa samples had additional heterochromatic bands in the centromeric region of the metacentric chromosomes and on the short arm of seven acrocentric chromosome pairs (Figure 1C).

Fluorochrome staining also showed divergent patterns among samples from different geographic regions. In the samples from Valença, we observed 13 bright $\mathrm{CMA}_{3}{ }^{+}$bands in diploid metaphases (Figure 2A). In the 9th and 12th chromosome pairs, these bands appear as large GC-rich blocks. Heteromorphisms were observed, in which one of the homologues showed differences in length (chromosome pairs 13,14) or even presence/absence of $\mathrm{CMA}_{3}$ 
A.

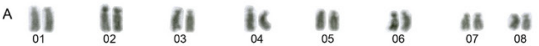

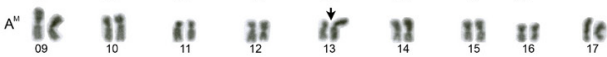

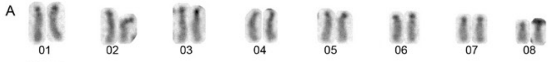

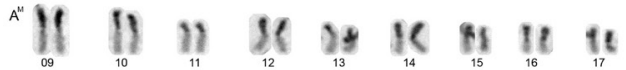

B.

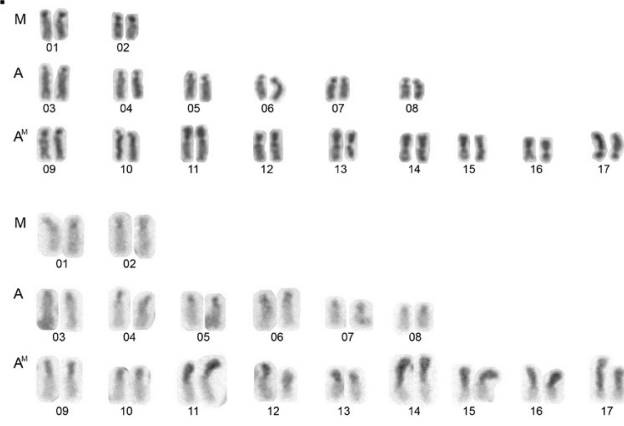

C.

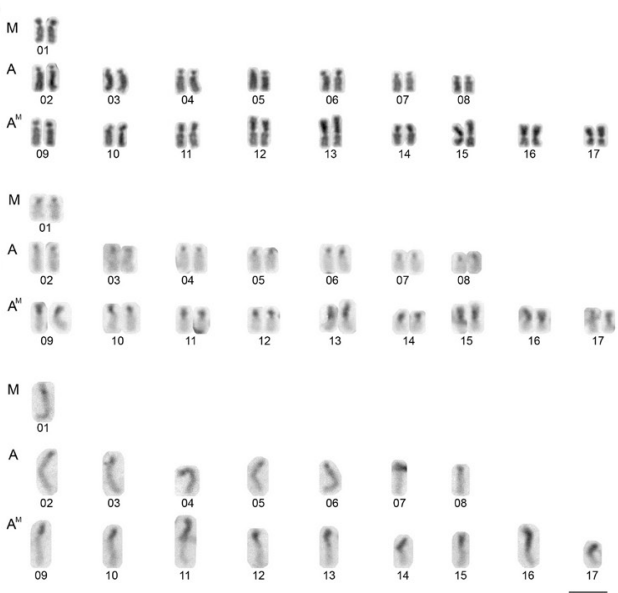

Figure 1. Conventional Giemsa staining and C banding in Scaptotrigona xanthotricha karyotypes showing: A. Females from Valença, BA, with $2 \mathrm{n}=34$ chromosomes; B. Females from Camacan, BA, with $2 \mathrm{n}=34$ chromosomes, and $\mathbf{C}$. Females and male, from Viçosa, $M G$, with $2 n=34$ and $n=17$ chromosomes, respectively. The arrow indicates heteromorphism in the 13th pair. The bar represents $10 \mu \mathrm{m}$.

bands (chromosome pair 17). Additionally, the 15th and 16th pairs had small telomeric bands. In the Camacan samples, we observed $12 \mathrm{CMA}_{3}{ }^{+}$bands in diploid metaphases. The 11 th, 12 th, 13th, and 15th pairs showed large blocks over one of the chromosome arms. Pair 16 was also heteromorphic and the 17th had a small telomeric band (Figure 2B). 


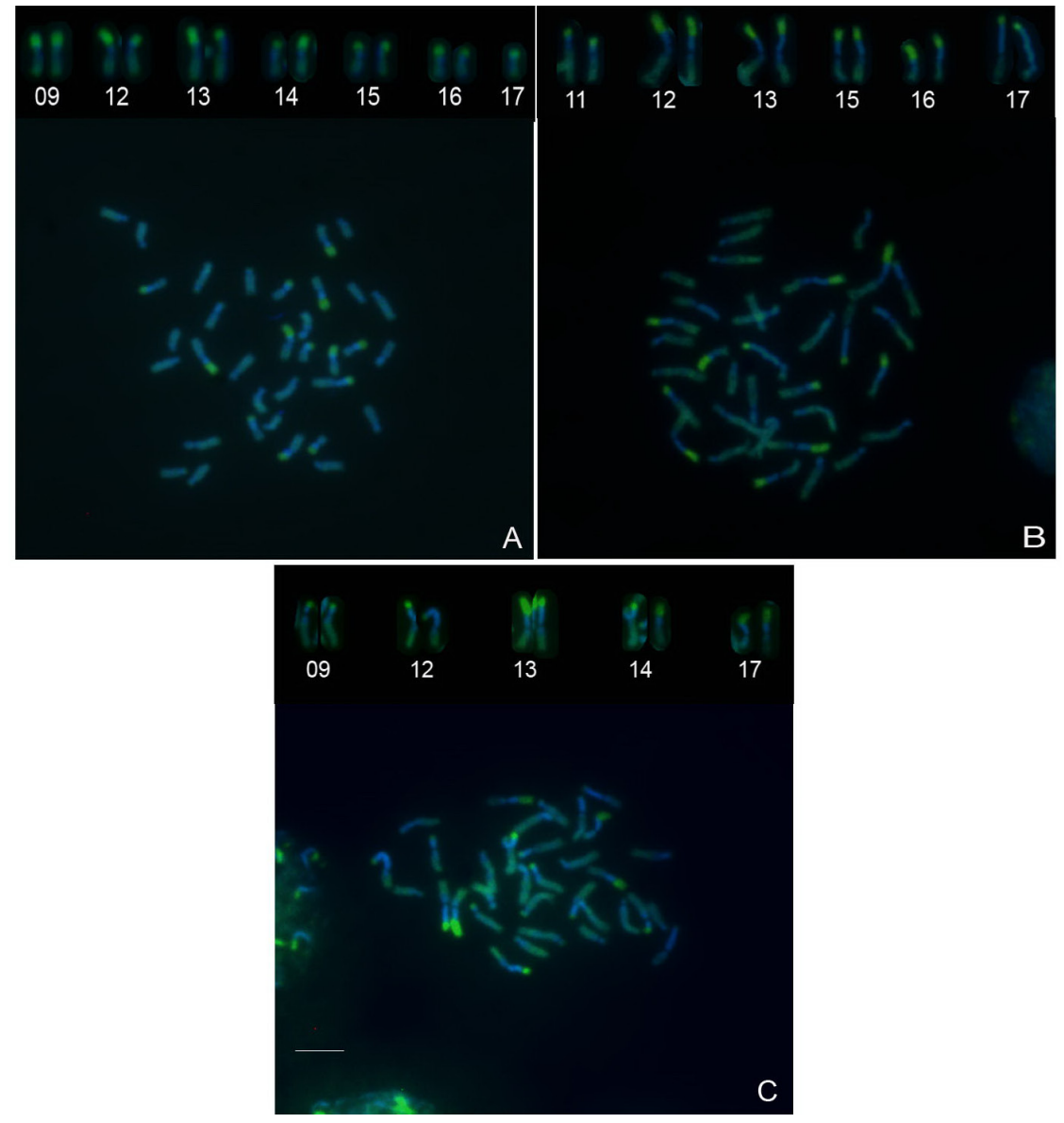

Figure 2. $\mathrm{CMA}_{3}$ /DAPI banding in metaphases of Scaptotrigona xanthotricha from: A. Valença, B. Camacan, and C. Viçosa populations. $\mathrm{CMA}_{3}$-stained chromosome pairs are shown in detail at the top of each metaphase. The bar represents $10 \mu \mathrm{m}$.

In the Viçosa samples, we observed $10 \mathrm{CMA}_{3}{ }^{+}$bands per diploid metaphase. The bands appear as small telomeric bands in the 9th and 12th pairs and as large continuous telomeric blocks in 13th, 14th, and 17th pairs. However, bands in the 13th and 14th pairs were heteromorphic (Figure 2C). DAPI-negative regions coincided with heterochromatic $\mathrm{CMA}_{3}^{+}$ blocks except for the pericentromeric regions.

Similarly to the results obtained with the previously used techniques, Ag-NOR banding showed variation in NOR length and location in the chromosomes. In the samples from Valença, we observed 12 Ag-NOR bands per diploid chromosome set (Figure 3A), most of which coincided in shape and location with the $\mathrm{CMA}_{3}{ }^{+}$bands. However, the 6th chromosome pair had a telomeric Ag-NOR band, which was not stained with $\mathrm{CMA}_{3}$ on the long arm. Alternatively, the 14th and 15 th pairs showed $\mathrm{CMA}_{3}{ }^{+}$staining not related to the Ag-NOR band. Pair 17th was homomorphic for Ag-NOR banding. 


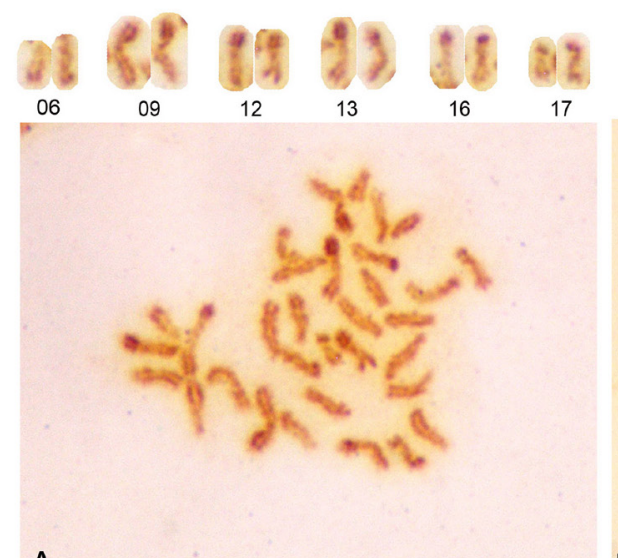

A

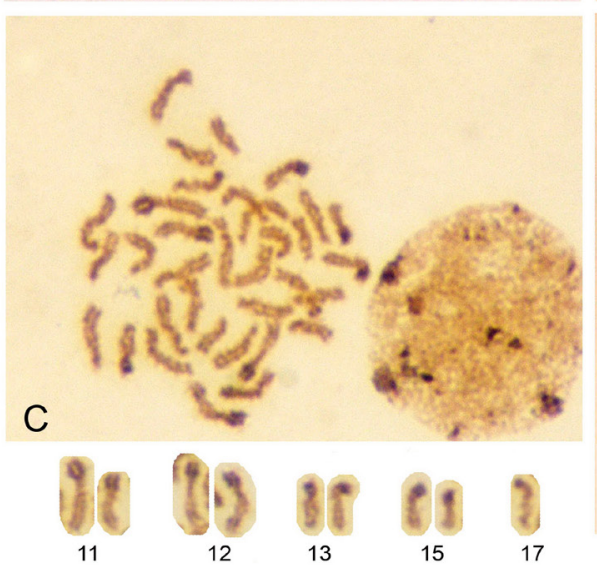

B
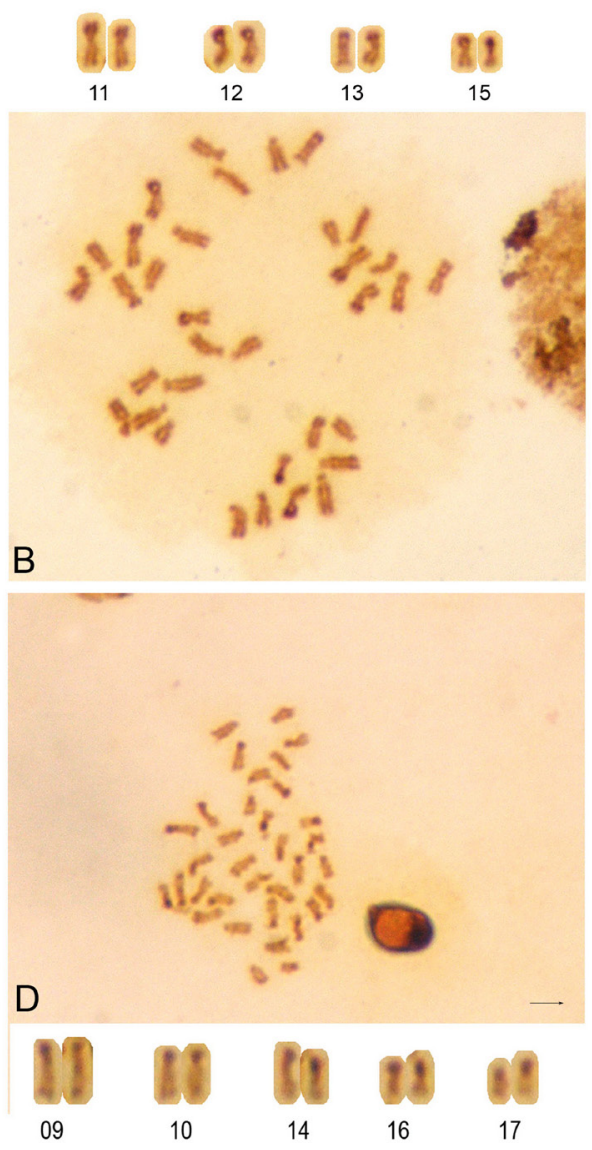

Figure 3. Ag-NOR staining in Scaptotrigona xanthotricha metaphases of: A. Sample from Valença, showing 12 bands, B. and C. Samples from Camacan, showing eight and nine bands, respectively, and D. Sample from Viçosa, showing ten bands. Ag-NOR-stained chromosome pairs are shown in detail at the top (A,B) or below (C,D) of each metaphase plate. The bar represents $10 \mu \mathrm{m}$.

Variation from eight to nine Ag-NOR bands per diploid set was observed in the Camacan samples (Figure 3B and $\mathrm{C}$ ). The NORs coincided with $\mathrm{CMA}_{3}^{+}$staining in most chromosomes, except for the 16th pair, which showed only $\mathrm{CMA}_{3}^{+}$bands (Figure 3B). In these samples, the heteromorphism in the 17th pair was due to the presence/absence of a minute telomeric Ag-NOR band (Figure 3C).

In the Viçosa samples, we found $10 \mathrm{Ag}-\mathrm{NOR}$ bands per diploid chromosome set, most of which coincided with $\mathrm{CMA}_{3}$ staining, except for chromosome pairs 12 and 13, which had only $\mathrm{CMA}_{3}^{+}$bands, and pairs 10 and 16, which showed only Ag-NOR bands (Figure 3D).

Most silver staining coincided with both $\mathrm{CMA}_{3}^{+}$bands and heterochromatic regions, revealing a predominant NOR location in GC-rich and heterochromatic chromosome regions. 


\section{DISCUSSION}

The occurrence of intraspecific karyotype variation has been described in different groups of Hymenoptera. However, most previous studies showed numeric variation as the main source of polymorphism (Brito et al., 1997; Araújo et al., 2002; Scher and Pompolo, 2003; Brown Jr. et al., 2004). The chromosome number $2 \mathrm{n}=34$ determined for Scaptotrigona xanthotricha agrees with previous studies on other Scaptotrigona species (Hoshiba and Imai, 1993; Rocha et al., 2003) and corroborates the numerical stability in the tribe Meliponini (Brito et al., 2003; Rocha et al., 2003; Costa et al., 2004).

We found interpopulational variation in heterochromatin content and distribution, location and number of GC and AT-rich blocks, and location of NOR bands. These results also differed from a previous karyotype description for this species by Rocha et al. (2003).

S. xanthotricha karyotype had a high and polymorphic heterochromatin content. Different techniques confirmed the existence of heteromorphism in the 13th chromosome pair in the samples from Valença, BA. This remarkable difference in the size of the short arm was also observed in the 14th pair by Rocha et al. (2003) in samples from near Viçosa, MG.

In Hymenoptera, heterochromatin heteromorphism was observed in previous studies on the wasp Trypoxylon (Trypargilum) nitidum (Scher and Pompolo, 2003), and on the bees Partamona helleri (Brito et al., 1997) and Friesella schrottkyi (Mampumbu AR, unpublished results).

Differential deletion, translocation, unequal crossover, and heterochromatin tandem growth have been suggested as possible causes of heterochromatin heteromorphisms (John, 1988; Hatanaka et al., 1998). According to Eichler and Sankoff (2003), these repetitive segments are hot spots for the occurrence of chromosomal rearrangements. Since these are faster evolving regions, the accumulation of this type of chromatin in the chromosomes of S. xanthotricha was an important element for the interpopulational differences found.

The multiple NOR polymorphism found in S. xanthotricha is congruent with heterochomatin variation, as most NOR bands overlapped with $\mathrm{C}$ bands. The three populations sampled showed geographic variation in number (from 8 to 12 bands per diploid genome), length and location of NORs.

This is the first report of multiple NORs in bees. In other bee species, only two NORs per diploid genome were found, e.g., Melipona (Maffei et al., 2001; Rocha et al., 2002); Friesella (Mampumbu AR, unpublished results), and Partamona (Brito et al., 2003), showing that this feature is uncommon in this group.

Heterogeneity in number and location of $\mathrm{CMA}_{3}$ bands among populations of S. xanthotricha was consistent with their geographic distribution. Numeric variation ranged from 10 to 13 bands, with the lowest number found in the samples from southeastern Brazil, Viçosa, MG. Samples of Camacan and Valença, both in the State of Bahia, northeastern Brazil, showed 12 and 13 bands, respectively. A maximum of 8 bands was previously reported in Viçosa and the nearby region by Rocha et al. (2003). Our results also suggest a northward increment in the number of GC-rich blocks in the genome of the species. Most $\mathrm{C}$ bands, NOR and $\mathrm{CMA}_{3}$ bands were congruent, which confirms the predominance of GC base pairs around the NORs mentioned by Sumner (2003) and agrees with similar results found in the bees Partamona peckolti (Brito et al., 2003) and Melipona asilvae (Rocha et al., 2002).

Fixed chromosome changes can establish different lineages that represent the first stages in the speciation process (King, 1993). Cytogenetic analyses have revealed the occur- 
rence of species complexes in several other groups of organisms (Crossa et al., 2002; Van Daele et al., 2004; Ravaoarimanana et al., 2004; Vicari et al., 2005; Rossi et al., 2005; Tinni et al., 2007). Our data raise questions about the presence of additional taxa in the extensively geographically distributed $S$. xanthotricha. Additionally, our results indicate a gradient of polymorphisms across the species' geographic range, with a consequent and larger cytogenetic differentiation between samples at both extremes of the distribution. Although the species shows nesting preference for the understory layer habitat in the Atlantic rainforest, there are environmental variations, for instance in temperatures and elevations, across this biome. Viçosa, MG, for example, is at about $600 \mathrm{~m}$ above sea level, while Valença, BA, is at sea level. The selective nesting behavior of this species combined with the recent fragmentation of the Atlantic rainforest certainly contributes to reduction in gene flow among the populations, which can enhance species differentiation.

Our karyotypic data using different cytogenetic techniques revealed a geographic distinction among the three S. xanthotricha populations. Further molecular analyses and larger sampling are being carried out for a better understanding of the phylogeographic structure of S. xanthotricha. Future reproduction studies in this species will also be helpful in evaluating the impact of chromosome variation on the taxonomic status of the species.

\section{ACKNOWLEDGMENTS}

We thank Dr. Gabriel A.R. Melo from the Universidade Federal do Paraná, Curitiba, for the identification of the species and to Dr. Janisete G. Silva-Miller and Dr. Fernanda A. Gaiotto for helpful comments on the manuscript. Research supported by FAPESB (Fundação de Amparo à Pesquisa do Estado da Bahia).

\section{REFERENCES}

Araújo SM, Silva CC, Pompolo SG, Perfectti F, et al. (2002). Genetic load caused by variation in the amount of rDNA in a wasp. Chromosome Res. 10: 607-613.

Brito RM, Costa MA and Pompolo SG (1997). Characterization and distribution of supernumerary chromosomes in 23 colonies of Partamona helleri (Hymenoptera, Apidae, Meliponinae). Braz. J. Genet. 20: 185-188.

Brito RM, Caixeiro APA and Pompolo SG (2003). Cytogenetic data of Partamona peckolti (Hymenoptera, Apidae, Meliponini) by $\mathrm{C}$ banding and fluorochrome staining with DA/CMA 3 and DA/DAPI. Genet. Mol. Biol. 26: 53-57.

Brown KS Jr, Von Schoultz B and Suomalainen E (2004). Chromosome evolution in Neotropical Danainae and Ithomiinae (Lepidoptera). Hereditas 141: 216-236.

Costa KF, Brito RM and Miyazawa CS (2004). Karyotypic description of four species of Trigona (Jurine, 1807) (Hymenoptera, Apidae, Meliponini) from the State of Mato Grosso, Brazil. Genet. Mol. Biol. 27: 187-190.

Crossa RP, Hernandez M, Caraccio MN, Rose V, et al. (2002). Chromosomal evolution trends of the genus Panstrongylus (Hemiptera, Reduviidae), vectors of Chagas disease. Infect. Genet. Evol. 2: 47-56.

Eichler EE and Sankoff D (2003). Structural dynamics of eukaryotic chromosome evolution. Science 301: 793-797.

Guerra M and Souza MJ (2002). Como Observar Cromossomos: Um Guia de Técnicas em Citogenética Vegetal Animal e Humana. FUNPEC Editora, Ribeirão Preto.

Hatanaka T, Tambasco AJ and Galetti-Junior PM (1998). Heterochromatin heterogeneity and chromosome heteromorphism in Cerdocyon thous (Mammalia, Canidae). Genet. Mol. Biol. 21: 227-231.

Hoshiba H and Imai HT (1993). Chromosome evolution of bees and wasps (Hymenoptera, Apocrita) on the basis of C-banding pattern analyses. Jpn. J. Entomol. 61: 465-492.

Howell WM and Black DA (1980). Controlled silver-staining of nucleolus organizer regions with a protective colloidal developer: a 1-step method. Experientia 36: 1014-1015.

Imai HT (1991). Mutability of constitutive heterochromatin (C-bands) during eukaryotic chromosomal evolution and their 
cytological meaning. Jpn. J. Genet. 66: 635-661.

Imai HT (1993). A theoretical approach to chromosome banding pattern analysis. Jpn. J. Genet. 68: 97-118.

Imai HT, Taylor RW, Crosland MW and Crozier RH (1988). Modes of spontaneous chromosomal mutation and karyotype evolution in ants with reference to the minimum interaction hypothesis. Jpn. J. Genet. 63: 159-185.

John B (1988). The Biology of Heterochromatin. In: Heterochromatin Molecular and Structural Aspects (Verma RS, ed.). Cambridge University Press, Cambridge, 1-128.

Kavalco KF and Pazza R (2004). A rapid alternative technique for obtaining silver-positive patterns in chromosomes. Genet. Mol. Biol. 27: 196-198.

King M (1993). Species Evolution: the Role of Chromosome Change. Cambridge University Press, Cambridge.

Maffei EM, Pompolo SG, Silva-Junior JC, Caixeiro AP, et al. (2001). Silver staining of nucleolar organizer regions (NOR) in some species of Hymenoptera (bees and parasitic wasp) and Coleoptera (lady-beetle). Cytobios 104: 119-125.

Mantovani M, dos Santos Abel LD, Mestriner CA and Moreira-Filho O (2000). Accentuated polymorphism of heterochromatin and nucleolar organizer regions in Astyanax scabripinnis (Pisces, Characidae): tools for understanding karyotypic evolution. Genetica 109: 161-168.

Moure JS, Urban D and Melo GAR (2007). Catalogue of Bees (Hymenoptera, Apoidea) in the Neotropical Region. Sociedade Brasileira de Entomologia, Curitiba.

Ravaoarimanana IB, Tiedemann R, Montagnon D and Rumpler Y (2004). Molecular and cytogenetic evidence for cryptic speciation within a rare endemic Malagasy lemur, the Northern Sportive Lemur (Lepilemur septentrionalis). Mol. Phylogenet. Evol. 31: 440-448.

Rocha MP, Pompolo SD, Dergam JA, Fernandes A, et al. (2002). DNA characterization and karyotypic evolution in the bee genus Melipona (Hymenoptera, Meliponini). Hereditas 136: 19-27.

Rocha MP, Pompolo SG and Campos LAO (2003). Citogenética da Tribo Meliponini (Hymenoptera, Apidae). In: Apoidea Neotropica: Homenagem aos 60 anos de Jesus Santiago Moure (Melo GAR and Alves-dos-Santos I, eds.). Editora UNESC, Criciúma, 311-320.

Rossi AR, Gornung E, Sola L and Nirchio M (2005). Comparative molecular cytogenetic analysis of two congeneric species, Mugil curema and M. liza (Pisces, Mugiliformes), characterized by significant karyotype diversity. Genetica 125: 27-32.

Scher R and Pompolo SG (2003). Evolutionary dynamics of the karyotype of the wasp Trypoxylon (Trypargilum) nitidum (Hymenoptera, Sphecidae) from the Rio Doce State Park, Minas Gerais, Brazil. Genet. Mol. Biol. 26: 307-311.

Schweizer D (1976). Reverse fluorescent chromosome banding with chromomycin and DAPI. Chromosoma 58: 307-324.

Sumner AT (1972). A simple technique for demonstrating centromeric heterochromatin. Exp. Cell Res. 75: 304-306.

Sumner AT (2003). Chromosomes: Organization and Function. Blackwell Publishing, Malden, Oxford, Melbourne, Berlin.

Tinni SR, Jessy NS, Hasan MM, Mustafa MG, et al. (2007). Comparative karyotype analysis with differential staining in two forms of Anabas testudineus bloch. Cytologia 72: 71-75.

Van Daele PAAG, Dammann P, Meier JL, Kawalika M, et al. (2004). Chromosomal diversity in mole-rats of the genus Cryptomys (Rodentia: Bathyergidae) from the Zambezian region: with descriptions of new karyotypes. J. Zool. 264: 317-326.

Vicari MR, Artoni RF and Bertollo LAC (2005). Comparative cytogenetics of Hoplias malabaricus (Pisces, Erythrinidae): a population analysis in adjacent hydrographic basins. Genet. Mol. Biol. 28: 103-110. 